\title{
Liquid Cell TEM Study of Nucleation and Growth of Dendrites
}

Matthew Hauwiller ${ }^{1}$, Wenjing Zheng ${ }^{1,2}$, Zhiyuan Zeng ${ }^{1}$, and Haimei Zheng ${ }^{1,3}$

${ }^{1}$ Materials Sciences Division, Lawrence Berkeley National Laboratory, Berkeley, CA, United States.

${ }^{2}$ Institute of New-Energy Materials, School of Materials Science and Engineering, Tianjin University, Tianjin, China

3. Department of Materials Science and Engineering, University of California, Berkeley, CA, United States.

The study of liquid phase reactions is significant for various applications including solution based materials synthesis, solar to fuel conversion, batteries and so on. By developing and applying in situ liquid environmental electron microscopy (TEM), we study nucleation, growth and materials transformations in liquids. Liquid cells with various designs, including those with $\mathrm{SiN}_{\mathrm{x}}$ windows, integrated electrodes or graphene, have been made. Many dynamic phenomena associated with the nucleation, growth and materials transformations have been revealed with high spatial and temporal resolution. We report the liquid cell TEM study of dendritic growth with or without an electric bias.

Dendritic growth has attracted lots of attention for many years [1-5] due to the complexity characterized as multilevel branching as well as the resulting device failures in modern technologies. As we know dendritic growth is ubiquitous in materials solidification and crystallization, which arises from the instabilities when growth rate is limited by the diffusion of ions from solution to the deposits. Real time observation of the nucleation and growth of dendrites with high spatial resolution give the opportunity to elucidate the dendrite formation mechanisms. For example, we study iron oxide dendritic growth in a liquid cell. By tracking the dendrite nucleation and growth trajectories, the branching mechanisms resulting from local composition fluctuations and growth kinetics dependent morphology development have been achieved (Figure 1).

Nucleation and growth of dendrites have also been observed at electrode-electrolyte interfaces in an electrochemical liquid cell. We have previously observed $\mathrm{Pb}$ dendrite formation in an aqueous electrolyte containing Pb precursor (Figure 2a) [6]. The lithium metal dendritic growth (Figure 2b) has also been observed in situ using a similar electrochemical liquid cell set up [7]. Recently, we have observed unique phenomena of $\mathrm{Na}$ deposition (Figure 2c) and have been able to resolve SEI layer effects. An understanding of dendritic formation of alkali metal has significance in modern technology such as high energy capacitors and batteries. Our in situ study shed lights on strategies of improving electrode design to reduce short-circuit failure and to enhance the performance of batteries [8].

References:

[1] W. A. Tiller, Science 146 (1964), p. 871.

[2] J. S. Langer, Science 243 (1989), p. 1150.

[3] Y. Miyata, M. E, Glicksman, S. H. Tirmizi, Journal of Crystal Growth 110 (1991), p. 683.

[4] K. G. Libbrecht, V. M. Tanusheva, Physical Review Letters 81 (1998), p. 176.

[5] T. Börzsönyi et al, Physical Review Letters 83 (1999), p. 2853.

[6] M. Sun et al, Sci Rep 3 (2013), p. 3227.

[7] Z. Y. Zeng et al, Nano Lett. 14 (2014), p. 1745. 
[8] Zheng acknowledges the funding support from U.S. Department of Energy (DOE), Office of Science, Office of Basic Energy Sciences, Materials Sciences and Engineering Division under Contract No. DE-AC02-05-CH11231 within the KC22ZH program.

Figure 1. (a) Formation of iron oxide dendrites in a liquid cell. (b) Tracking the development of dendrites by self-developed MATLAB codes.
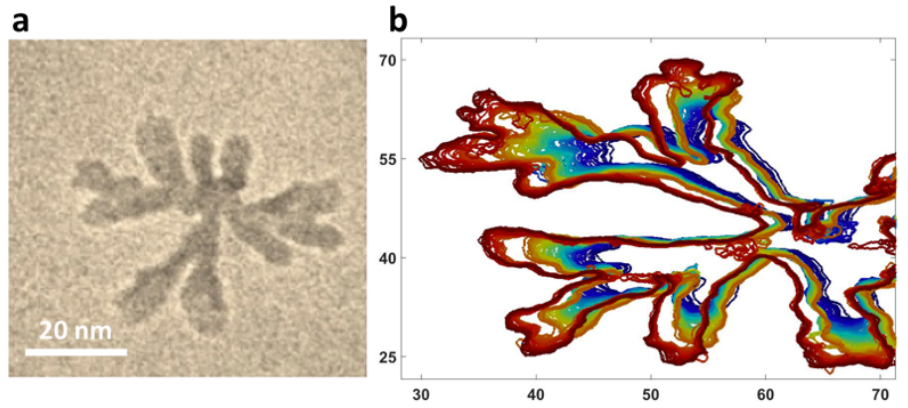

Figure 2. (a) Sequential TEM images from a movie show the growth of $\mathrm{Pb}$ dendrites. (b) Nucleation and growth of lithium dendrites. (c) Evolution of $\mathrm{Na}$ deposition on the shape Ti electrode in an electrochemical liquid cell.

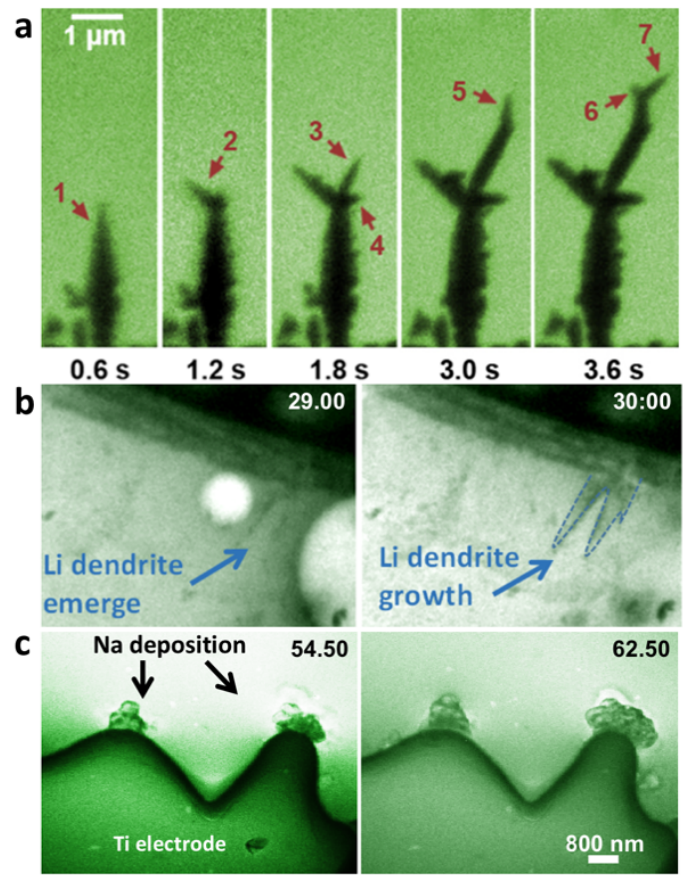

